\title{
Toroidal Variable-Line-Space Gratings: The Good, the Bad and the Ugly
}

\author{
Edward West ${ }^{1}$, Ken Kobayashi ${ }^{2}$, Jonathan Cirtain ${ }^{1}$, Allen Gary ${ }^{2}$, \\ John Davis ${ }^{1}$ and Joseph Reader ${ }^{3}$ \\ ${ }^{1}$ Space Science Office, VP62, NASA Marshall Space Flight Center, Huntsville, AL 35812 \\ ${ }^{2}$ The University of Alabama, Huntsville, CSPAR, Huntsville, AL 35899 \\ ${ }^{3}$ National Institute of Standards and Technology, Gaithersburg, MD 20899
}

\begin{abstract}
Toroidal variable-line-space (VLS) gratings are an important factor in the design of an efficient VUV solar telescope that will measure the CIV $(155 \mathrm{~nm})$ and MgII $(280 \mathrm{~nm})$ emissions lines in the Sun's transition region. In 1983 Kita and Harada described spherical VLS gratings but the technology to commercially fabricate these devices is a recent development, especially for toroidal surfaces. This paper will describe why this technology is important in the development of the Solar Ultraviolet Magnetograph Investigation (SUMI) sounding rocket program (the good), the delays due to the conversion between the TVLS grating design and the optical fabrication (the bad), and finally the optical testing, alignment and tolerancing of the gratings (the ugly).
\end{abstract}

Keywords: UV polarization, Solar Magnetograph, Sun, Sounding Rocket, Toroidal gratings, TVLS gratings

\section{INTRODUCTION}

The Solar Ultraviolet Magnetograph Investigation, SUMI, has been reported in several papers since this program began in $2000^{8,9,10}$. The emphasis of this paper is to describe SUMI's Toroidal Variable-Line-Space (TVLS) gratings. These gratings help SUMI meet its scientific goals which require both high spectral resolution and high optical efficiency for magnetic field measurements in the vacuum ultraviolet wavelength band of the solar spectrum (the good). Unfortunately, the technology readiness level of these gratings has made their implementation difficult, especially for a sounding rocket payload (the bad). Therefore, this paper emphasizes the problems and solutions that were developed to use these gratings in SUMI (the ugly).

Section 2 contains a short review of the scientific goals of SUMI and why this mission is important in the understanding of the 3D structure of the magnetic field on the Sun. The flight hardware that makes up the SUMI payload is described in Section 3 with emphasis on those components that affect the TVLS gratings. Section 4 emphasizes the alignment, testing and optical modeling that were developed to optimize the performance of these gratings.

\section{SCIENCE GOALS}

This section summarizes the importance $e^{1,2}$ of direct magnetic field measurements at various heights in the solar atmosphere and how the SUMI sounding rocket program plans to verify that transition region magnetic field measurements are possible.

\subsection{Magnetic field measurements in the transition region}

The problem of how energy, stored in the solar magnetic field, is released to heat the corona and drive the dynamic phenomena of the outer atmosphere, flares and coronal mass ejections, remains unsolved. Neither theoretical or numerical models nor current observations are able to provide a conclusive story. We have learned that a substantial fraction of this energy propagates outward into the interplanetary medium in the form of EM radiation, energetic 
particles, and the solar wind. These constitute space weather and have been shown to affect the immediate geo-space environment and create hazards for terrestrial and space assets. In the future, space weather will be a major concern for astronauts working on the lunar surface and traveling through interplanetary space. The solar input is the starting point for any model that describes and predicts space weather. Therefore understanding the processes that contribute to both the steady and transient release of magnetic energy into the solar atmosphere is a crucial element of these studies.

To understand the fundamental processes that lead to the explosive release of magnetic energy in solar flares and coronal mass ejections (CMEs), it is necessary to observe and infer the topology of the magnetic field and how it evolves prior to and during the energy release process. Success has been achieved in the characterization of the full vector field in the photosphere, where $\beta$, the ratio of the gas pressure to the magnetic pressure, is $>1$. At higher levels in the atmosphere (Figure 1$)^{3}$ where $\beta<1$, the magnetic field, through the Lorentz force, controls the structure and dynamics of the solar atmosphere, and rapid changes in its structure can produce energetic events. However, observations of the magnetic field at these higher levels are difficult, placing a serious limitation on our understanding of the physical processes occurring there.

The missing element is the ability to understand and describe quantitatively the transition layer where the field is no longer pressure-dominated as in the photosphere. The need to understand the energy release processes was identified two decades $\mathrm{ago}^{4}$, and was reaffirmed as the major conclusion of a workshop on the definition of the scientific goals for "Beyond Solar-B" (Moore, Davis, and Hathaway, 2001) . To understand this process, full vector magnetic field measurements at several different heights in the solar atmosphere, from the photosphere to the upper transition region where the field becomes nearly force free, are required. The vector field in the photosphere and low chromosphere has been measured with ground- based instruments. Spacebased instruments such as the Michelson Doppler Interferometer (MDI) on the Solar \& Heliospheric Observatory (SoHO) have demonstrated the importance of continuous, distortion free, observations of the longitudinal magnetic field from space to in our understanding of the Sun's magnetic field. The Solar Optical Telescope (SOT) on the Hinode and the Helioseismic and Magnetic Imager (HMI) on the Solar Dynamics Observatory (SDO) spacecraft will extend our space based observations to include photospheric vector magnetic fields. Although these high resolution observations will greatly advance our knowledge of the behavior of the magnetic field in the photosphere, they will shed little light on how the magnetic field higher in the solar atmosphere transitions to a nearly force-free
Table 1: This table shows a comparison of candidate lines that are being developed for current and future space-based photospheric, chromospheric and transition region vector magnetic field measurements.

\begin{tabular}{|c|c|c|c|c|c|}
\hline $\begin{array}{c}\text { Spectral } \\
\text { lines }\end{array}$ & $\begin{array}{c}\lambda \\
(\mathrm{nm})\end{array}$ & $\mathrm{g}$ & $\begin{array}{c}\lambda^{2} \times \mathrm{g} \\
\times 10^{4}\end{array}$ & $\begin{array}{c}\text { Height } \\
(\mathrm{km})\end{array}$ & Remarks \\
\hline \multicolumn{7}{|c|}{ Transition Region } \\
\hline CIV & $\mathbf{1 5 5 . 0}$ & $\mathbf{1 . 2}$ & $\mathbf{2 . 9}$ & $\mathbf{2 2 0 0}$ & SUMI \\
\hline MgII & $\mathbf{2 8 0 . 0}$ & $\mathbf{1 . 2}$ & $\mathbf{9 . 4}$ & $\mathbf{2 0 0 0}$ & SUMI \\
\hline \multicolumn{7}{|c|}{ Chromosphere } \\
\hline CaII & 854.2 & 1.1 & 80.2 & 1319 & \\
\hline NaI & 589.5 & 1.3 & 45.1 & 700 & Photosphere \\
\hline \multicolumn{7}{|c|}{} \\
\hline FeI & 630.2 & 2.5 & 99.2 & 250 & Hinode \\
\hline
\end{tabular}

MTRAP $^{6}$ : The Magnetic Transition Region Probe has all of these lines in its baseline mission.

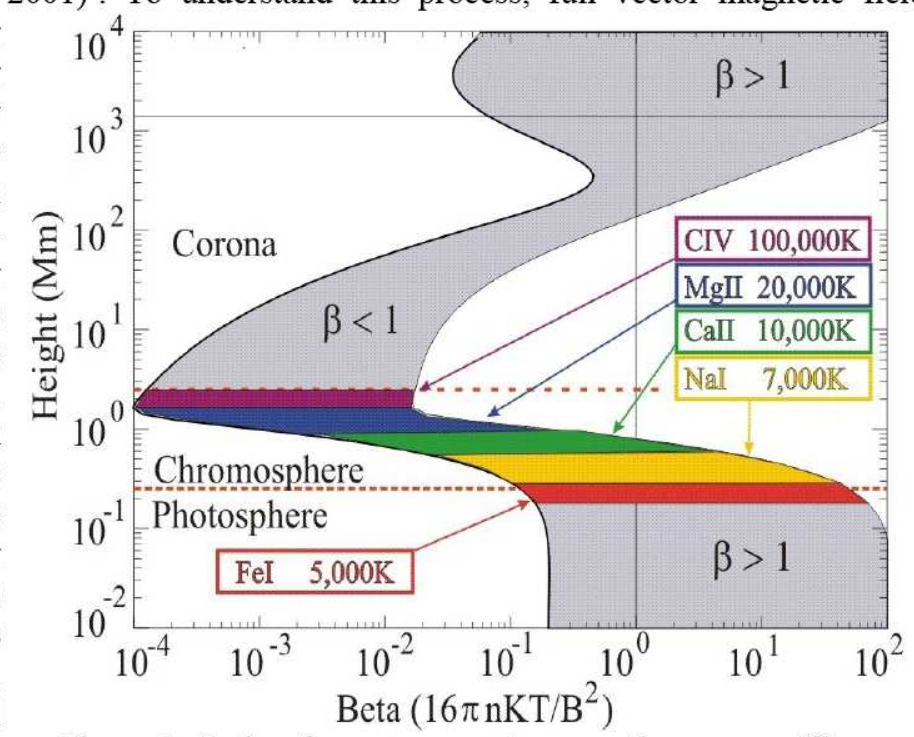

Figure 1. Ratio of gas pressure to magnetic pressure $(\beta)$ as a function of height, for an assumed range of photospheric magnetic field strength of 100 to $2500 \mathrm{G}$ (Gary, 2001) . $^{3}$. 
state. This requires measurement of the field using spectral lines that are formed at temperatures above $20,000{ }^{\circ} \mathrm{K}$. The two spectral lines we have chosen for SUMI are $280 \mathrm{~nm}$ in $\mathrm{MgII}$ and $155 \mathrm{~nm}$ in CIV. These lines occur in the far ultraviolet and are inaccessible from the ground. Together with the lines FeI, NaI and CaII, they form a quintet of magnetically sensitive lines chosen for a future mission called the Magnetic Transition Region Probe (MTRAP) ${ }^{6}$. The demonstration that the MgII and CIV lines can meet the requirements for the force-free region is a prerequisite for this mission and is one of the objectives of the Solar Ultraviolet Magnetograph Investigation (SUMI).

\subsection{Goals for SUMI sounding rocket program}

Measuring CIV has always been the driving force in the development of SUMI. This line is formed in the relatively thin transition region which simplifies its interpretation. However, the magnetic sensitivity, $\lambda^{2} \mathrm{~g}$ where $\lambda$ is the wavelength and $g$ is the Landé $g$ factor, is low compared to lines in the visible and infrared. Table 1 compares the SUMI lines with lines that are being used in photospheric and low chromospheric magnetographs. Certainly the results from SUMI will impact future missions as scientist try to understand the 3-D structure of the Sun's magnetic field.

\section{DESCRIPTION OF SOUNDING ROCKET PAYLOAD}

The Solar Ultraviolet Magnetograph Investigation (SUMI) began as a set of development programs to improve the efficiency of polarization measurements in the ultraviolet. This section describes the sounding rocket payload with emphasis on those components that affect the Toroidal Variable-Line-Space (TVLS) gratings.

As demonstrated by Table 1, polarization measurements in the ultraviolet require high spectral resolution due to the small magnetic sensitivity $\left(\lambda^{2} \mathrm{~g}\right)$. Higher spectral resolution is required to resolve the Zeeman splitting. Therefore to reduce the length of the telescope while increasing the spectrograph (with improved resolution), a cold mirror RitcheyChretien telescope was chosen over a traditional Gregorian telescope (\$3.1). To improve the photon and polarization efficiency, a $\mathrm{MgF}_{2}$ double Wollaston polarizer was selected as the analyzer so that simultaneous measurements of orthogonal polarizations could be made (\$3.2). Section 3.3 will describe the Toroidal Variable-Line-Space (TVLS) grating technology that is used to achieve the high spectral resolution while reducing the number of optical elements. The last section (\$3.4) describes SUMI's cameras.

\subsection{Telescope optics}

A solar telescope design must solve the thermal problems associated with direct solar viewing. The simplest solution is a Gregorian telescope with a field stop between the primary and secondary mirrors. While this reduces the thermal load on the secondary by rejecting the unwanted light, the disadvantages are its limited field of view, a longer optical path, and a larger secondary for on-axis designs, which decreases the effective collecting area of the telescope. SUMI's approach for decreasing the thermal load on the secondary mirror is to use a Ritchey-Chretien telescope with special dielectric coatings applied to the front surfaces of both the primary and secondary mirrors (Figure 2). These coatings reflect only the narrow wavelength ranges around the CIV and MgII emission lines which results in a "cold mirror", i.e., a "self-filtering" telescope. The rear surface of the primary mirror is figured and has an aluminum coating that reflects the unwanted radiation back through the telescope and into space. The advantages of this design are that the field of view is not restricted (the whole Sun can be imaged) and, for a given instrument length, the smaller telescope size allows a larger spectrograph improving the wavelength resolution. The narrowband UV reflection coatings simplify the thermal environment, minimize infrared and visible light contamination of the spectral data, and act as a blocking filter for the spectrograph. The measured reflectance of these coatings is shown in Figure 3. Although the MgII bandpass may appear off-center, its reflectivity was tuned to minimize the $1^{\text {st }}$ order contamination of $3100 \AA$ light into the CIV measurement, which is made in $2^{\text {nd }}$ order.

\subsection{Polarimeter and slit jaw camera}

The focal plane optics following the telescope includes the polarimeter, spectrograph slit and slit jaw camera. The polarimeter consists of a $\mathrm{MgF}_{2}$ waveplate and a $\mathrm{MgF}_{2}$ double Wollaston analyzer. Due to the low photon flux at CIV 


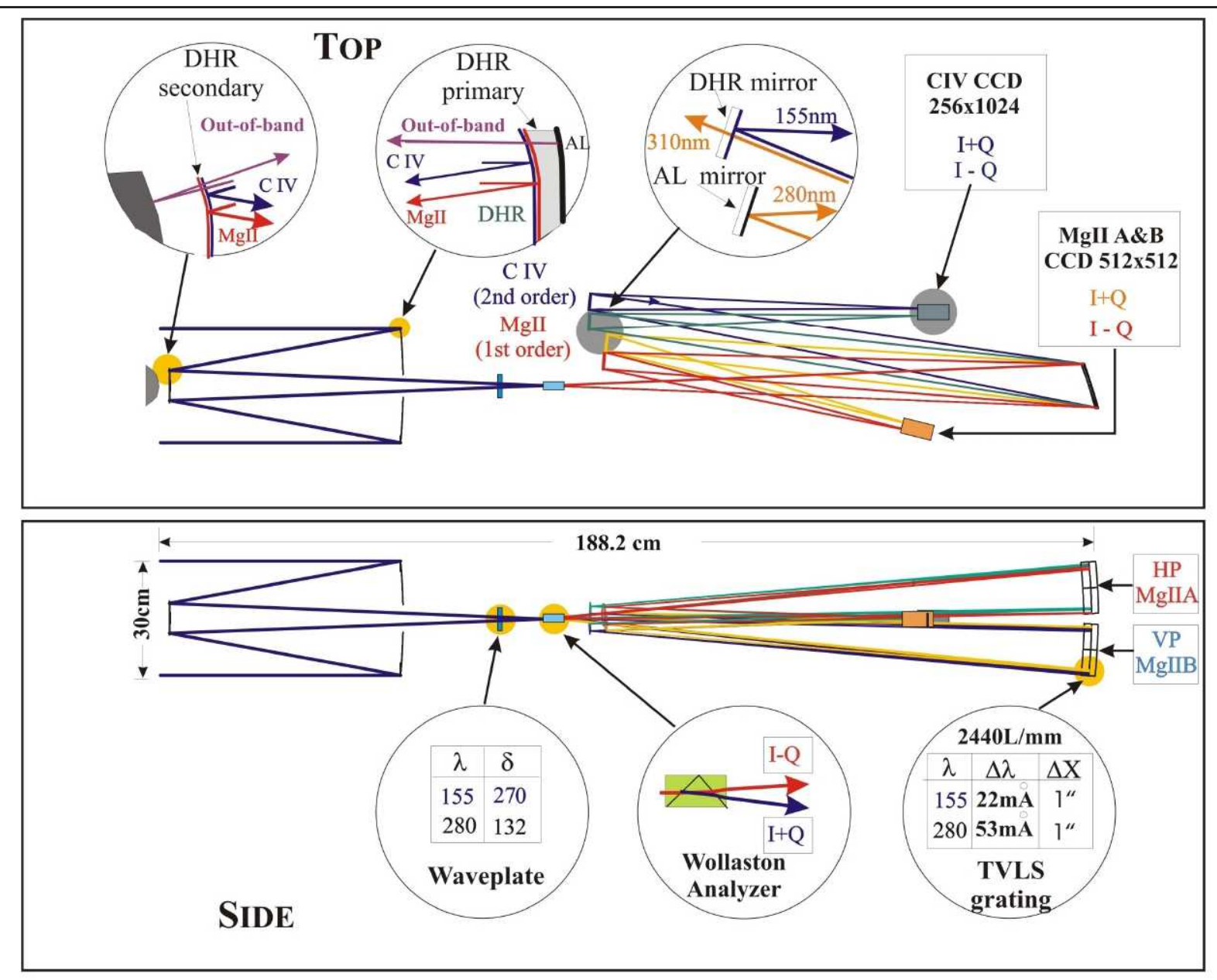

Figure 2. Optical schematic showing the technologies developed in the SUMI sounding rocket program. The CIV wavelength is $155 \mathrm{~nm}(1550 \AA)$ and the $\mathrm{MgII}$ is $280 \mathrm{~nm}(2800 \AA)$. The side view shows the double Wollaston analyzer and the two TLVS gratings designated by the I+Q/VP and I-Q/HP beam paths. Two frame transfer $512 \times 512$ cameras are used in the $\mathrm{MgII}$ measurement (MgIIA/HP and MgIIB/VP). A single full frame 256x1024 CCD is used for the CIV VP/HP measurements.

and the weak linear polarization levels, the waveplate was designed to measure circular polarization at CIV (270 retardance at $1550 \AA)$ and the full Stokes vector at $\mathrm{MgII}\left(132^{\circ} \text { retardance at } 2800 \AA\right)^{9,11}$. During the flight SUMI will concentrate on circular polarization measurements when the payload is above $200 \mathrm{~km}$ (required height for CIV measurements) and will make the linear polarization measurements when the sounding rocket is below $200 \mathrm{~km}$.

The double Wollaston analyzer which is a polarizing beamsplitter is the most efficient UV polarizer in this wavelength band $^{9}$. Figure 4 shows the orientation of the double Wollaston and the exiting linearly polarized beams. The orientation of the linear polarization and the polarization reflectivity of the TVLS gratings were discussed in a previous paper ${ }^{12}$. The selected gratings maximize the reflectance of the vertical linear polarization $(\mathrm{VP}=+\mathrm{Q}$ defined to be the $\mathrm{E}$ field aligned to the slit, spatial axis), and horizontal linear polarization ( $\mathrm{HP}=-\mathrm{Q}$ is perpendicular to slit, dispersion axis).

A dual-beam analyzer is very important in transition region magnetic field measurements since the CIV emission is subject to rapid changes in intensity ${ }^{18}$. Without simultaneous measurements of orthogonal polarizations, intensity crosstalk can create false signals in SUMI's magnetic field measurements. Since both polarizations are observed, the total transmission is much higher than traditional reflective polarizers even at CIV (the cutoff wavelength of $\mathrm{MgF}_{2}$ is 


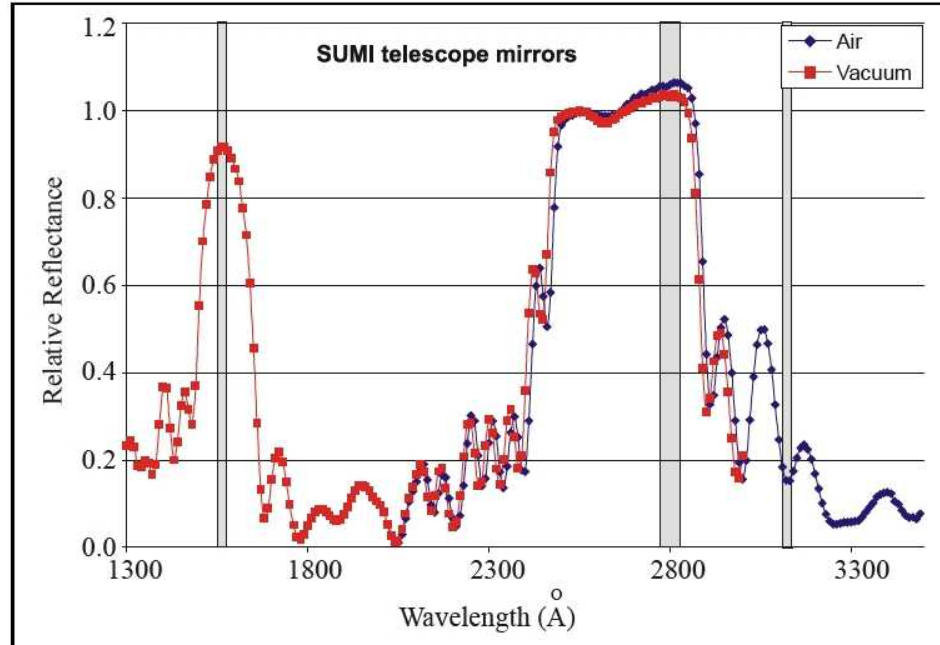

Figure 3. Dielectric High Reflectance (DHR) coating on SUMI primary and secondary telescope mirrors. These coatings act as a prefilters to isolate the CIV (1550 $\AA$ ) and MgII $(2800 \AA)$ lines. The MgII reflectivity was tuned to minimize the $1^{\text {st }}$ order reflectance of $3100 \AA$ into the $2^{\text {nd }}$ order CIV measurement.

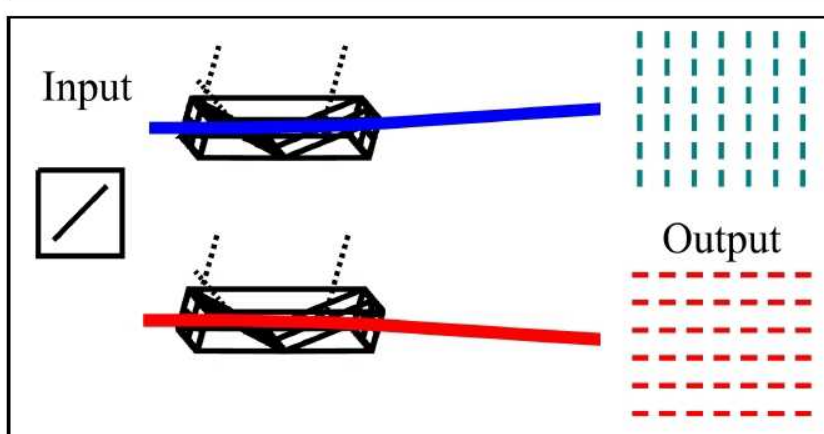
polarizations exiting the analyzer. The upper image is the polarization parallel to the slit (vertical polarization, VP); the lower image perpendicular to the slit (horizontal polarization, HP).
Figure 4. Orientation of the double Wollaston with the linear

$1150 \AA)$. Finally, the polarization resolution $\left(<10^{-}\right.$ $\left.{ }^{3}\right)$ is higher and covers a larger wavelength range than traditional reflective analyzers which is

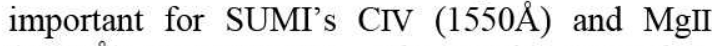
$(2800 \AA)$ measurements. The rotating waveplate allows cross-checks of the image subtraction scheme by flipping the polarization signals from the Sun between the HP3/VP5 detectors. For example, with the waveplate fast axis (FA) at $45^{\circ}$ the VP5 detector would measure I+V for CIV and the HP3 detector I-V; with the FA at $135^{\circ}$ VP5 would measure I-V and HP3 I+V (see Figure 7 for VP5/HP3 CIV detector definition).

The spectrograph slit has a unique structure. The slit is cut into a $6 \mathrm{~mm}$ thick diamond turned aluminum substrate and is $\sim 3.7 \mathrm{~mm}$ long (3.5 arc minutes) with $0.5 \times 0.5 \mathrm{~mm}(30 \mathrm{arcsec})$ boxes on each end. The width of the slit has been split into two sections, a $30 \mu \mathrm{m}(\sim 1.6$ arc seconds) and a $60 \mu \mathrm{m}(\sim 3.2$ arc second). Due to the exploratory nature of the SUMI measurements, the uncertainty in the target at launch and the limited observing time of the flight ( $\sim 6$ minutes), this structure was adopted to give SUMI a larger dynamic range and to ensure capturing enough photons to make meaningful polarization measurements at $\mathrm{CIV}$.

\subsection{Spectrograph}

Since the double Wollaston analyzer produces two polarized images of the slit, two toroidal variable-line-space (TVLS) gratings are required $^{13,14,15}$. The sensitivity of the spectrograph gratings to the polarization exiting the double Wollaston was taken into consideration ${ }^{8}$. In our initial concept a quarterwave plate was placed between the double Wollaston prism and gratings to convert the exiting linear polarization to circular if the gratings were found to be very sensitive to linear polarization. Fortunately, a bare aluminum TVLS grating can be used for the vertical polarization (VP) and a $\mathrm{MgF}_{2}$ coated aluminum grating for the horizontal polarization (HP see Table 2). Section 4 discusses both the optical modeling and measurements made on these devices.

\subsection{UV cameras}

The SUMI cameras are based on the E2V back illuminated, bare silicon technology. This technology enhances the UV quantum efficiency but at the time SUMI was developed their design was limited to certain CCD structures since they were not part of E2V's standard product line in 2005. For the slit-jaw camera, and the MgII cameras where high speed electronic shuttering was required, a frame transfer $512 \times 512 \mathrm{CCD}$ array with $13 \mu \mathrm{m}$ pixels was selected. The CIV camera uses a full frame $1024 \times 256 \mathrm{CCD}$ array with $26 \mu \mathrm{m}$ pixels. To simplify the electrical wiring between the cameras 
Table 2. Polarization reflectivity measurements for the six gratings fabricated by Jobin Yvon for SUMI: 2007 measurements. The gratings selected for the first flight are \#3 for the HP (HP3) and \#5 for the VP (VP5).

\begin{tabular}{|c|c|c|c|c|c||}
\hline \multirow{2}{*}{ Grating number } & \multirow{2}{*}{ Coating } & \multicolumn{2}{|c|}{ Horizontal Polarization (HP) } & \multicolumn{2}{|c|}{ Vertical Polarization (VP) } \\
\cline { 3 - 6 } & & $\begin{array}{c}\mathrm{CIV} \\
\left(2^{\text {nd }} \text { order }\right)\end{array}$ & $\begin{array}{c}\text { MgII } \\
\left(1^{\text {st }} \text { order }\right)\end{array}$ & $\begin{array}{c}\text { CIV } \\
\left(2^{\text {nd }} \text { order }\right)\end{array}$ & $\begin{array}{c}\text { MgII } \\
\left(1^{\text {st }} \text { order }\right)\end{array}$ \\
\hline $1 . \mathrm{CY} 11 \mathrm{~S}^{*}$ & $\mathrm{AL} / \mathrm{MgF}_{2}$ & $\mathbf{1 2 \%}$ & $\mathbf{3 9 \%}$ & $29 \%$ & $52 \%$ \\
\hline $2 . \mathrm{CY} 11 \mathrm{O}$ & $\mathrm{AL} / \mathrm{MgF}_{2}$ & $11 \%$ & $33 \%$ & $15 \%$ & $38 \%$ \\
\hline $3 . \mathrm{CY} 11 \mathrm{~N}^{*}$ & $\mathrm{AL} / \mathrm{MgF}_{2}(A L)$ & $\mathbf{1 2} \%(16 \%)$ & $\mathbf{4 1 \%}(9 \%)$ & $\mathbf{3 0 \%}(17 \%)$ & $\mathbf{5 4 \%}(49 \%)$ \\
\hline $4 . \mathrm{CY} 11 \mathrm{P}$ & $\mathrm{AL}$ & $16 \%$ & $11 \%$ & $18 \%$ & $52 \%$ \\
\hline $5 . \mathrm{CY} 11 \mathrm{R}$ & $\mathrm{AL}$ & $12 \%$ & $15 \%$ & $\mathbf{2 0 \%}$ & $\mathbf{6 2 \%}$ \\
\hline $6 . \mathrm{CY} 11 \mathrm{M}$ & $\mathrm{AL}$ & $14 \%$ & $16 \%$ & $17 \%$ & $61 \%$ \\
\hline
\end{tabular}

* Acton $\mathrm{AL} / \mathrm{MgF}_{2}$ coatings. Grating 3 was recoated by Acton after the aluminum measurements showed a low HP reflectance at $\mathrm{MgII}(9 \%)$

and the data system, a USB 2.0 interface is used. For ground-based testing and software development, a $\mathrm{MgF}_{2}$ window is mounted in front of all of the cameras. The measured quantum efficiency (QE) for these cameras is $>50 \%{ }^{17}$.

The SUMI sounding rocket has two data systems to control the four USB cameras, the rotating waveplate and to monitor the health and status of the SUMI payload. Each data system has two USB and two Ethernet ports on the processor board. Since the CIV measurements have the highest priority, the CIV data system is the master and drives the timing and telemetry for the SUMI instrument and controls the MgII data system. All of the data from SUMI's cameras is stored within the payload on a 4GB solid state disk drive attached to each data system. The slit jaw camera is connected to the second USB port on the CIV data system.

\section{DESIGN, FABRICATION AND TESTING SUMI'S TVLS GRATINGS}

Kita and Harada described the use of spherical VLS gratings in $1983^{13}$ and Thomas extended this technology to toroidal surfaces in $2003^{13}$. Due to the wavelength dispersion of the double Wollaston analyzer, a toroidal surface was an important development for SUMI's CIV and MgII measurements This section discuses the optical design and fabrication of the gratings ( $\$ 4.1$ ), and the optimization, modeling and testing (§4.2) of SUMI's TVLS gratings in the sounding rocket payload. The optical design and fabrication are grouped together because it took several iterations to develop an optical design that would fit within a sounding rocket. After the TVLS gratings were fabricated, their polarization properties were measured and two of the six gratings were selected for SUMI's first flight. The original set was grating 1 for the HP (HP1) and and grating 3 for VP (VP3). After a failure during vibration testing, a second set of gratings was selected, HP3 and VP5. The reason for this selection was based on the MgII HP reflectivity measurements which were higher for the aluminum gratings with a $\mathrm{MgF}_{2}$ protective coating (Table 2). The $\mathrm{MgF}_{2}$ overcoat reduces the loss in the CIV reflectivity due to the oxidation of the aluminum coating and, while there is some improvement in the CIV VP reflectivity, the bare aluminum gratings will be used for the VP measurements so that there is a backup $\mathrm{MgF}_{2} /$ AL HP grating.

\subsection{Design and Fabrication}

The SUMI design was developed and fabricated at different times: (1.) Telescope ${ }^{10}-2000$, (2.) Polarimeter ${ }^{9}-2001$ and (3.) TVLS gratings ${ }^{14}-2003$. This paper will only cover the design and fabrication of the TVLS gratings.

Certainly the development of the TLVS technology was good news for SUMI. With TVLS gratings SUMI could achieve its wavelength resolution while improving its optical efficiency with the elimination of relay optics in the spectrograph. The problem was that the TVLS technology was just being developed which made the transition between the optical design and the fabrication an educational process. This "educational" development increased the cost and created delays in the SUMI sounding rocket program. 


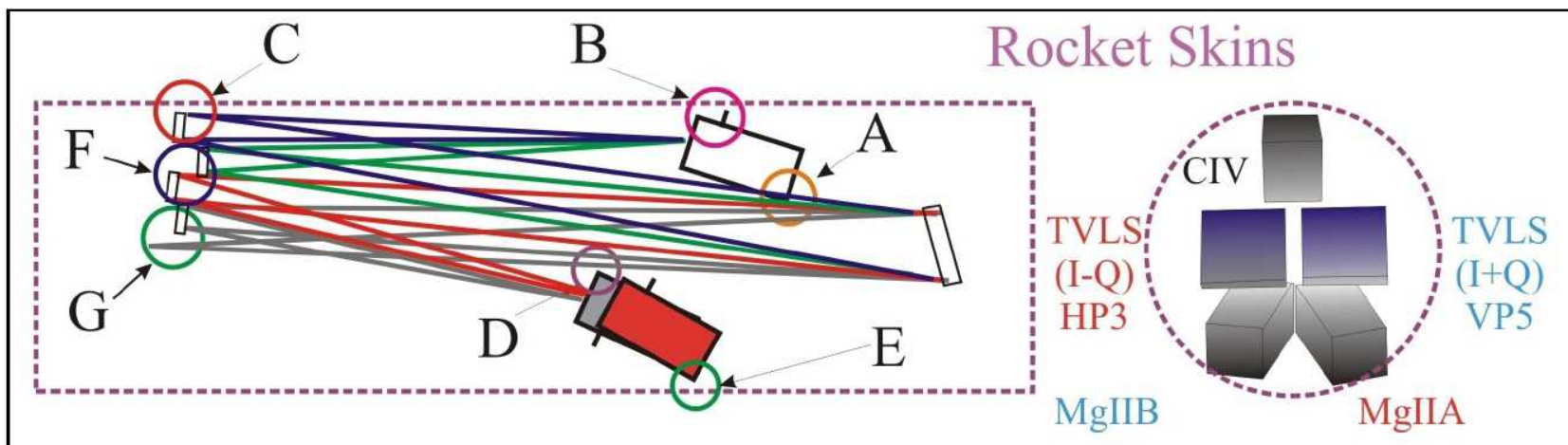

Figure 5. The critical areas in SUMI mechanical alignment that required adjustments to the original optical design. See Figure 6 for a 3D model of the SUMI spectrograph.

\subsubsection{Development of the SUMI Optical Design}

The TVLS gratings went through several optical design cycles. Since the fabrication of these gratings required a long lead time, the initial TVLS design was completed in July, 2003 so that the procurement could be awarded. As the fabrication began, the second phase required adjustments in the placement of the gratings and the fold mirrors in the SUMI payload. The first design change was the beam separation between the VP and HP optical paths. This adjustment required that the gratings be staggered so that there was sufficient separation between them that they would not come into contact with each other during the $10 \mathrm{G}$ vibration tests (Side view of Figure 2). The second adjustment to the optical design was positioning the fold mirrors. This optical design required an adjustment in the CIV HP3 and the MgII VP5 ( $\boldsymbol{F}$ in Figure 5) fold mirror positions which was achieved by a small difference in the tilt of the HP and VP gratings (y tilt in Figure 6). The placement of the CIV VP5 fold mirror within the payload (C) and the MgII HP3 fold mirror away from the beams exiting the double Wollaston $(\underline{G})$ also had to be considered. Finally, the USB interface of the flight cameras was longer than expected. That extra length coupled with the cooling ports ( $\underline{B}$ in Figure 5$)$ for the cameras thermal electric coolers required a final adjustment to the optical design to keep the cameras away from the rocket skins $(\underline{B} \boldsymbol{\&} \boldsymbol{E})$ while eliminating any vignetting of the beam between the double Wollaston and TVLS gratings $(\underline{A \& D})$. While some loss in resolution was expected as the optical design was adjusted, this resolution loss was small compared to the ruling errors encountered during the fabrication of the TVLS gratings (Figure 7, 2005 data).

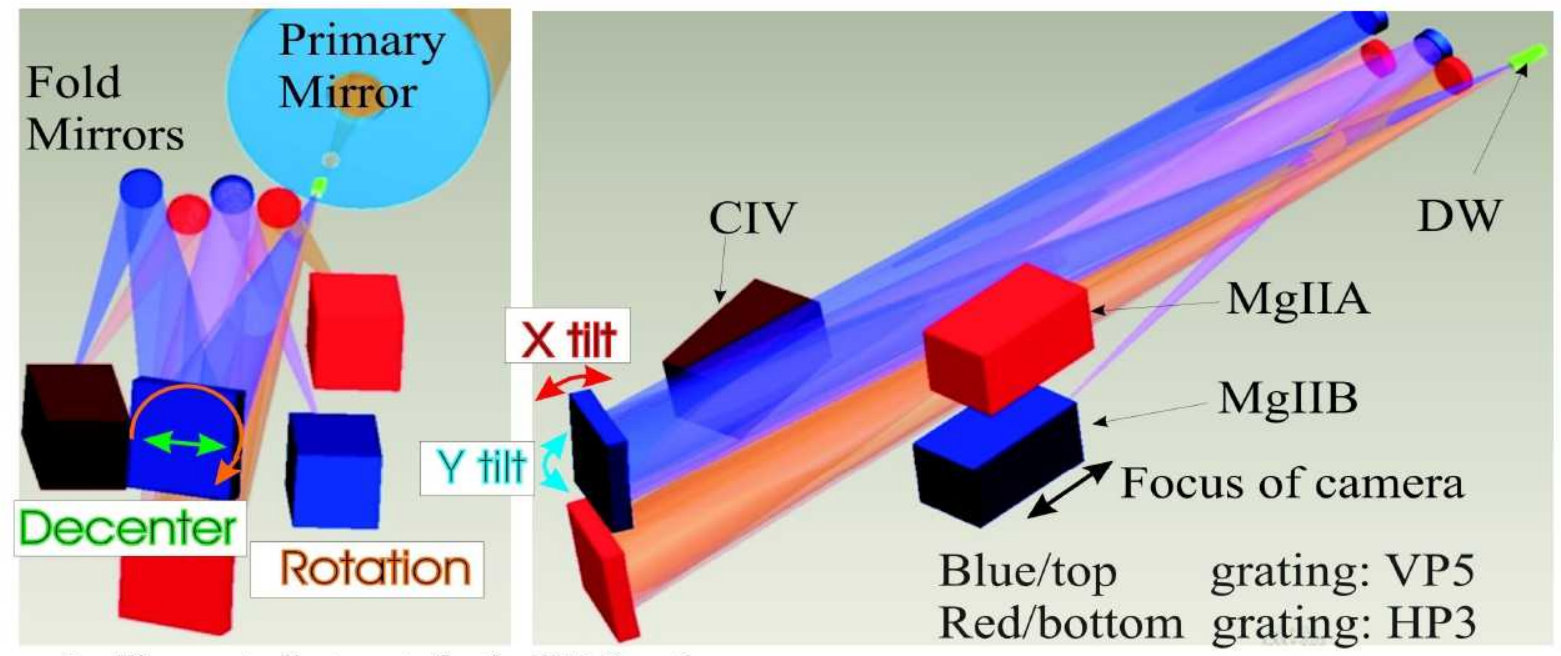

Figure 6. Alignment adjustments for the TVLS gratings 


\subsubsection{Fabrication}

To optimize the instrument's imaging performance while fitting it into a sounding rocket payload, the spectrograph was designed to measure CIV in $2^{\text {nd }}$ order and MgII in $1^{\text {st }}$ order. The two TVLS gratings use the same ruling equation. The ruling only varies by $\sim 2 \%$ around the central groove density of 2.440 lines per $\mu \mathrm{m}$ (Figure 7 ). These ruling variations can be described by a third-order polynomial with horizontal distance $(y)$ on the grating blank (in $\mathrm{mm}$ ):

$$
\rho(y)=\frac{1}{\frac{1}{\rho_{0}}+\alpha y+\beta y^{2}+\gamma y^{3}} \quad \text { (Zemax equation for VLS ruling) }
$$

where $\rho(y)$ is the ruling as a function of distance from center of the grating, and $\rho_{0}$ is the central ruling frequency (lines $/ \mu \mathrm{m}$ ). The ruling parameters for the SUMI gratings are: $\rho_{0}=2.440, \alpha=-1.2755 e-4, \beta=1.182 e-9$ and $\gamma=2.4729 e-11$. Both gratings have the same concave toroidal radii of curvature: $1500.000 \mathrm{~mm}$ in the dispersion axis and $1601.792 \mathrm{~mm}$ in the spatial direction.

In October 2004, the manufacturer shipped SUMI's gratings to the optics group at GSFC to verify their performance.

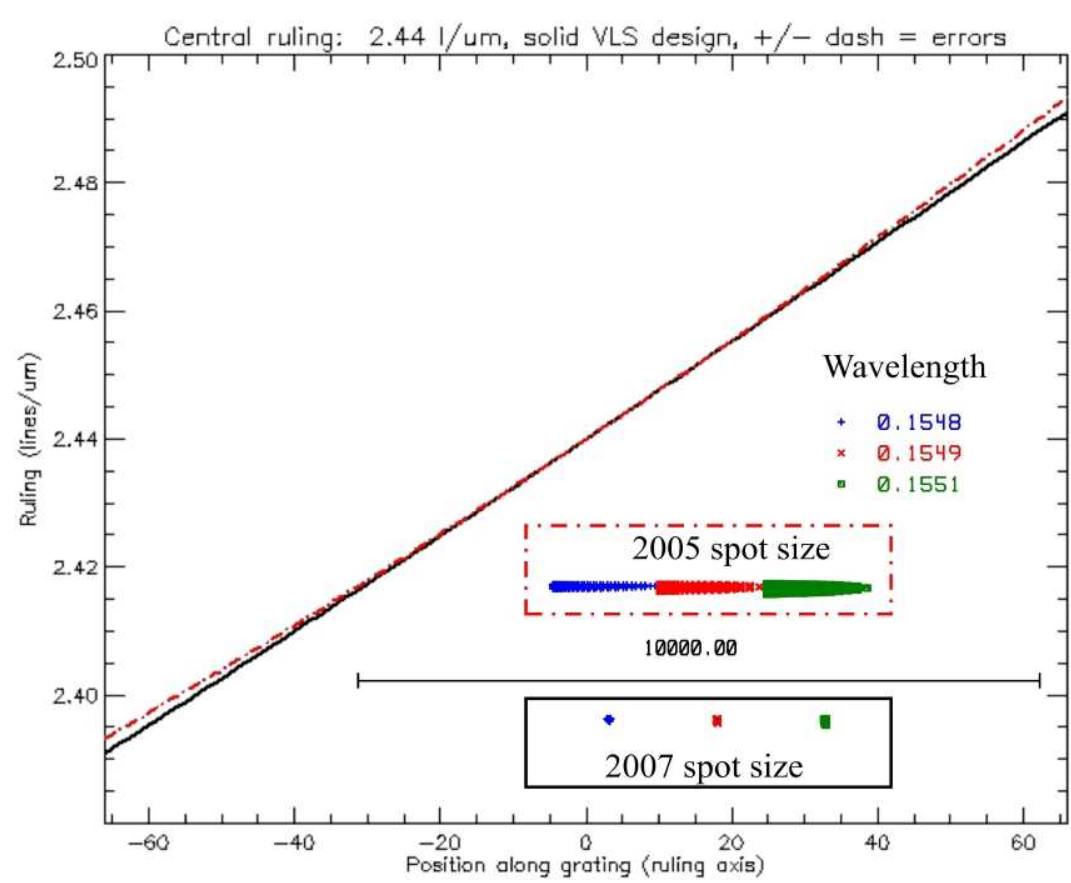

Figure 7. 2007 VLS ruling (black line, Zemax parameters: $\alpha=-1.2755 e-4, \beta=1.182 e-$ 9 and $\gamma=2.4729 e-11$.) and the 2005 VLS (red dashed line, $\alpha=-1.2714 e-4, \beta=-$ $8.8364 e-8$ and $\gamma=1.555 e-11)$.
While the curvatures of the toroidal surfaces were correct, an error in the translation of the Zemax ruling equation required that the gratings be re-ruled (Figure 7: original rulings, 2005 red dash curve). This required that the grating designer become very familiar with the manufacturer's holographic ruling equipment. After several iterations adjusting the ruling equations of the Zemax optical design and the ruling equipment, the TVLS gratings were re-ruled and finally delivered to MSFC August 2006 (Figure 7: 2007 black solid curve). During this redesign, an analysis study was done on the new Zemax ruling parameters which showed that $\alpha$ was the critical parameter in achieving SUMI's wavelength resolution. Since $\alpha$ is the linear coefficient in the ruling equation, the assumption was made that, if the manufacturer stayed within their error budget, a small decenter and tilt of the grating could compensate for that error.

\subsection{Testing and Modeling the SUMI spectrograph}

This section describes the development of a special VUV test facility that would interface to the SUMI sounding rocket and provide the spectral lamps needed to align the TVLS gratings (\$4.2.1)and the optical model to help in the alignment of the CIV and MgII lines onto the spectrograph cameras (§4.2.2). 


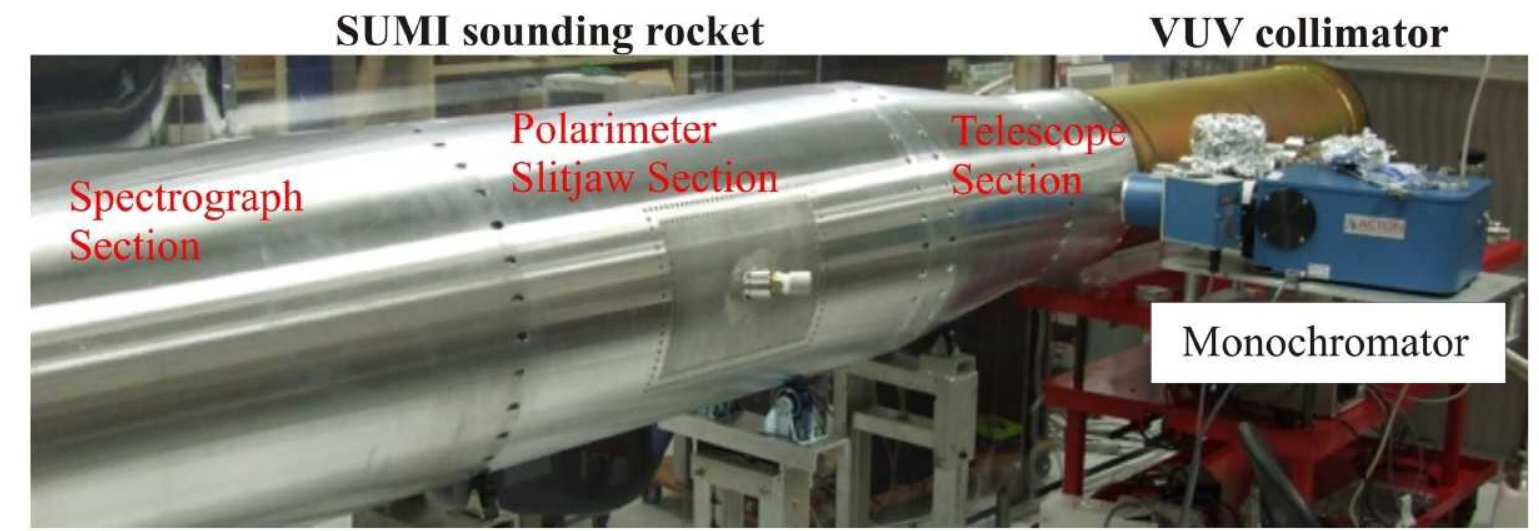

Figure 8. SUMI sounding rocket attached to the VUV collimator (gold telescope) with the Acton VM504. The VM504 was replaced with a Carbon lamp for the CIV alignment and a PtNe lamp for the MgII tests.

\subsubsection{VUV collimator - spectral alignment}

Since each optical element represents a loss in photons, especially at CIV, the goal for SUMI was to isolate the CIV and MgII lines using as few optics as possible. This approach led to the development of the dielectric high-reflectivity (DHR) coatings on the telescope mirrors (\$3.1, Figure 3) which act as a dual-line prefilter. While SUMI is efficient in isolating our UV lines, traditional methods to align the instrument cannot be used. Therefore a custom vacuum ultraviolet (VUV collimator) facility had to be developed that was within the budget constraints of our sounding rocket program.

Although the VUV collimator cannot achieve SUMI's resolutions, it can be aligned with traditional instruments. This collimator is an all reflecting system with aluminum $/ \mathrm{MgF}_{2}$ mirrors that are optimized for $120 \mathrm{~nm}$. After aligning the VUV collimator, it can be used to provide the vacuum UV wavelengths needed to check the alignment of the SUMI telescope and spectrograph.

Our original concept used an Acton VM504 monochromator for the spectral calibration of SUMI but SUMI's higher wavelength resolution (2440 lines/mm TVLS gratings compared to the 1200 line/mm Acton grating), the monochromator's $\pm 0.1 \mathrm{~nm}$ uncertainty in its wavelength drive and the uncertainty in the wavelength position of the Acton deuterium lamp's emission lines led to the decision to use a carbon spark lamp for the CIV alignment (Section 4.2.2) and the platinum neon (PtNe) lamp for the MgII (Section 4.2.3).

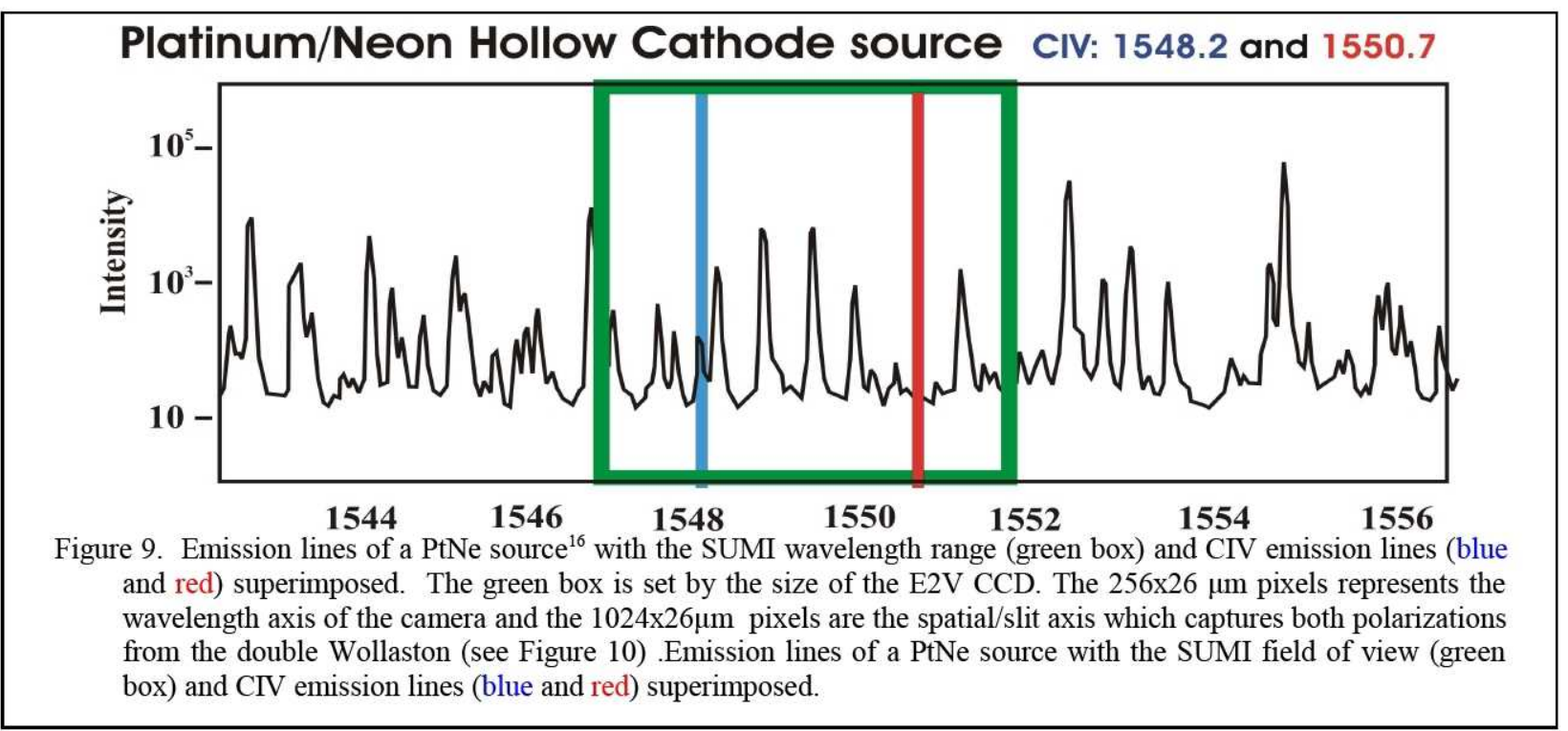




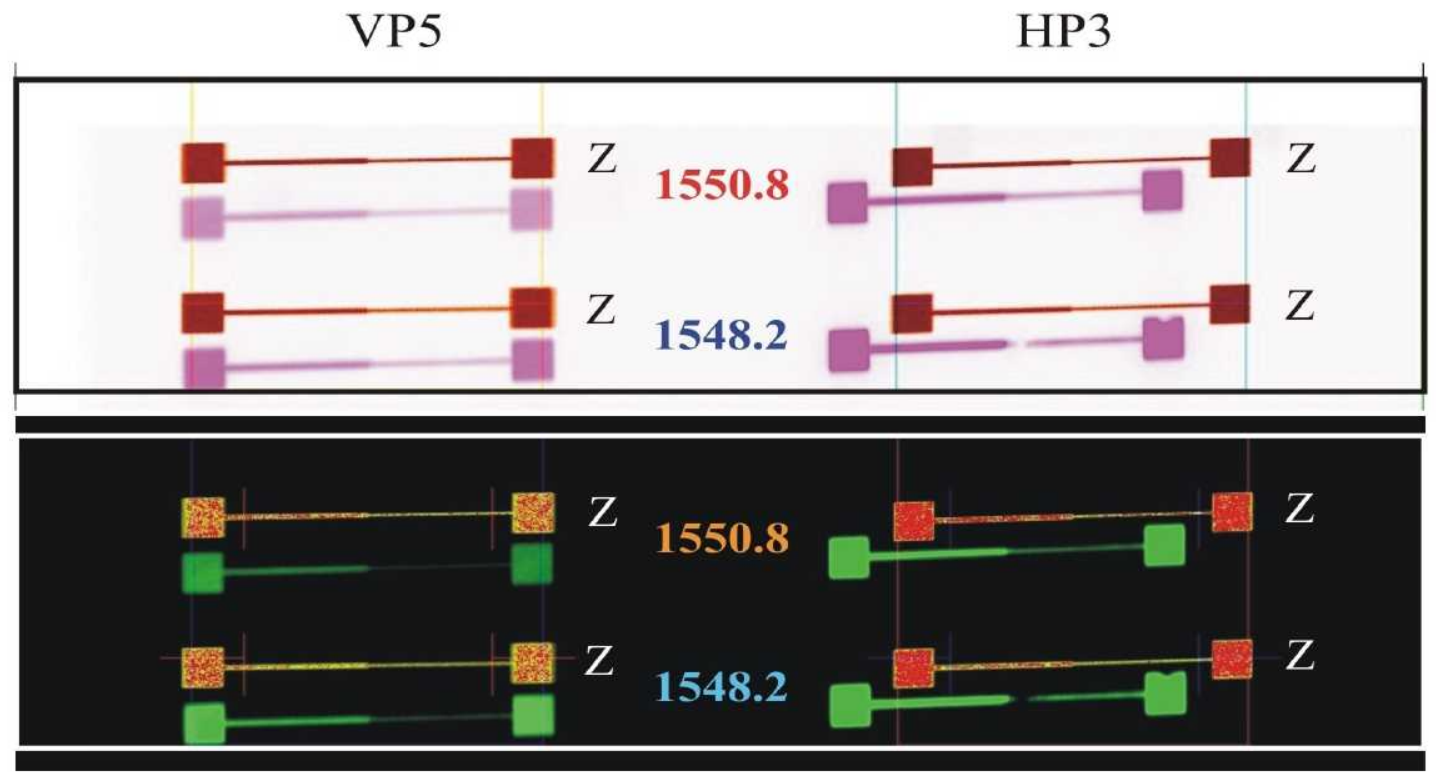

Figure 10. SUMI display screen showing the overlay of the Zemax spectra (Labeled Z) on the SUMI spectrograph cameras. The image on the top are inverted and is the same as the lower image. The CIV camera sees both the horizontal (HP3) and vertical (VP5) gratings (green and light purple) at the same time.

\subsubsection{CIV alignment}

The initial alignment of the gratings began with the MgII cameras and the PtNe source but the large number of lines led to some uncertainty in identifying the emission lines in the MgII wavelength band (Figure 11). While the CIV alignment requires a vacuum $\left(<10^{-3}\right.$ torr $)$, the carbon spark lamp actually provided the best wavelength calibration since it produced the CIV emission lines with only a few lines near that CIV wavelength band. With special wrenches, the tilt of the gratings were adjusted in a vacuum so that the CIV lines were centered onto the CIV camera.

To help in the alignment, a Zemax model was developed with the primary wavelengths near the CIV (and MgII) wavelength band and the spectrograph's custom slit. The weight parameter in the wavelength data was used to simulate the intensity differences of the various lines. A dummy line with a weight of 0.001 was created in the wavelength 1 position that was the midpoint between the CIV (and MgII) emission lines. With this setup, a Geometric Image Analysis (GIA) file could be created to show all of the emission lines. The field of view of the Zemax model was set up to be twice that of the spectrograph cameras so that nearby emission lines could be seen and compared to the images from the cameras.

Figure 10 shows an overlay of the Zemax model with the images from the CIV camera. In normal operation the CIV spectra are stored into the green memory plane of the display while the Zemax model is stored in the red and blue planes. Additional line plots (not shown) are used to align the CIV spectra onto to Zemax model. These locations are indicated by the lines added to the Zemax GIA image.

\subsubsection{MgII alignment}

With the alignment of the VP5/HP3 gratings near the correct position, the MgII alignment, which can be done in air, was accomplished by developing a Zemax model of the strongest PtNe lines near the MgII lines. Figure 11 shows the PtNe source in the MgII wavelength range. The lines that can be easily resolved in the MgII cameras are $>10^{4}$. The $\mathrm{MgII}$ lines (red and blue lines in Figure 11) are overlaid onto the PtNe spectrum with the green solid box indicating the 


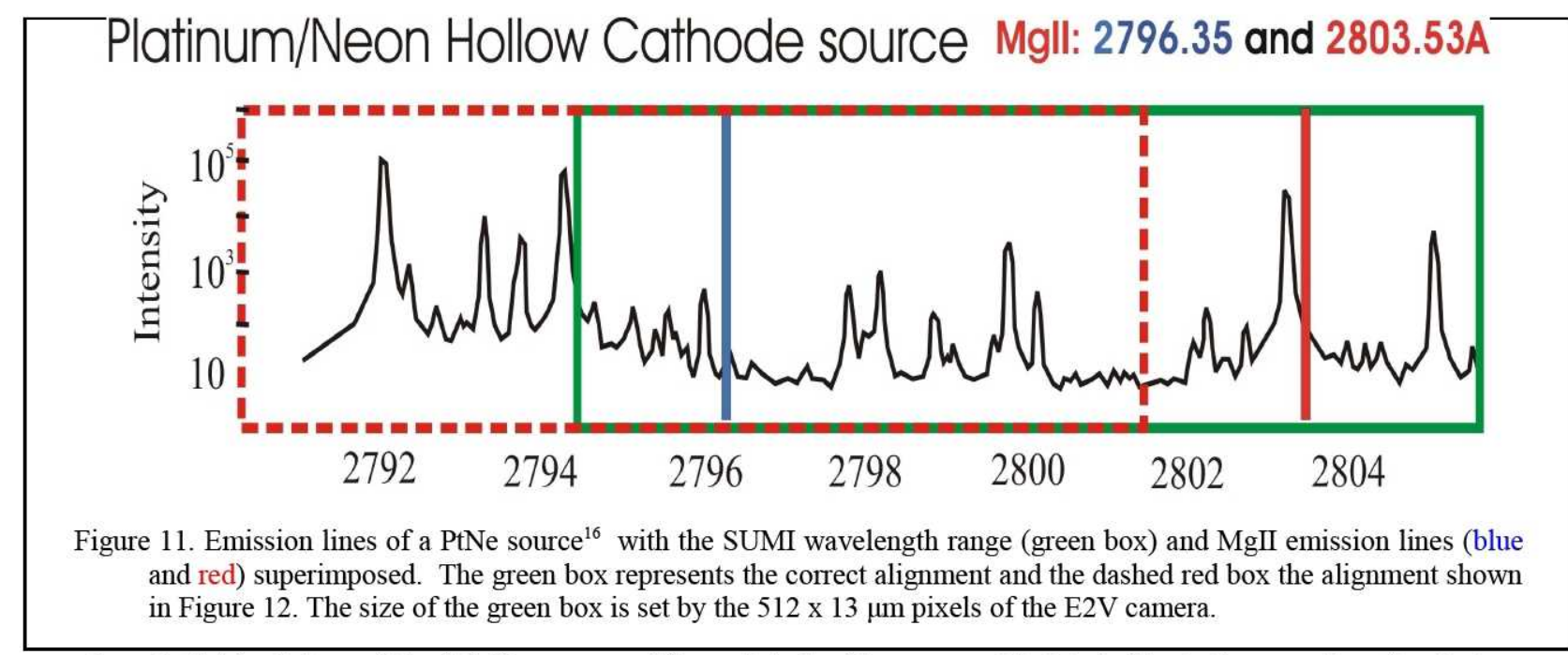

wavelength field of view of the MgII cameras. The red dashed box was added to indicate the wavelengths that are seen in Figure 12. Figure 12 shows the Zemax model with its alignment grid and the PtNe lines observed by the MgIIA (HP3) camera. When correctly aligned the right overlay plot will show "white" slit images (black on the right image). Since the Zemax model also tries to match the intensity of the PtNe lines (using the Weight parameter), the real-time test images will also be a shade of gray.

\section{SUMMARY}

TVLS gratings offer the designer the option to maximize the optical efficiency and wavelength resolution with a single optical component. This is especially important when making magnetic field measurements in the UV where every optical element represents a loss in the optical efficiency of the system. Unfortunately, there was a gap between the optical design tools that describe the variable-line rulings and the fabrication technology. This uncertainty must be understood and evaluated before manufacturing these gratings (3 year delay in our schedule). Although TVLS gratings

MgII: 2796.4 \& 2803.5 (2799.9)
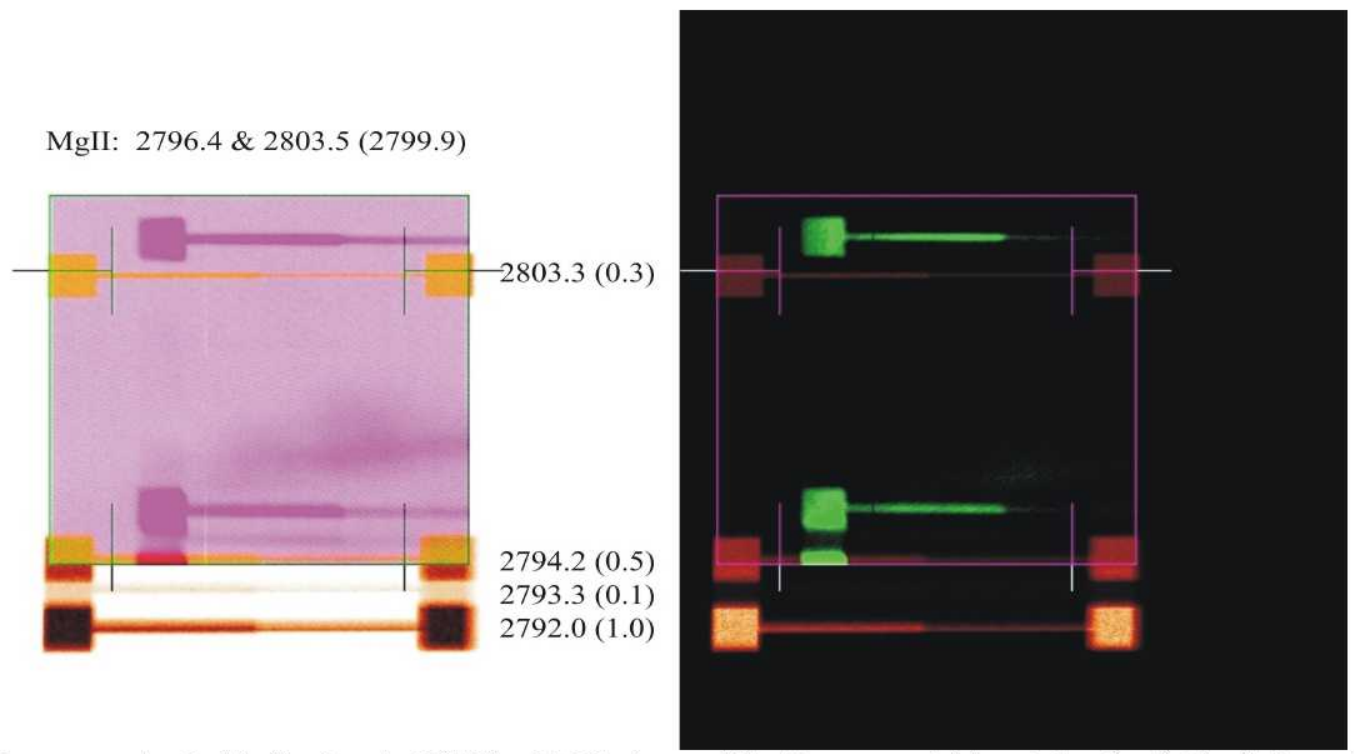

Figure 12. The Zemax spectra (red/yellow) onto SUMI's MgIIA image. The Zemax model has twice the field of view of the $\mathrm{MgII}$ camera. The image on the left is the negative of right image (normal mode) to allow identification of the weaker PtNe lines. The PtNe lines are between the two images and the MgII lines are listed in the top left image with the midpoint wavelength in parenthesis. 
are still custom devices, they are important for high resolution VUV spectral measurements and, as more of these devices are produced, the gap between fabrication and design should be reduced. While the packaging of the TVLS gratings within a sounding rocket payload was challenging and resulted in some loss in resolution, the budget, schedule, optical analysis tools and test equipment were the primary problems associated with aligning these gratings. While the CIV alignment went more smoothly than expected, the MgII slit images forced us to improve our optical models and reexamine our alignment procedures and tolerances to explain our MgII images especially in our vertical polarization tests.

\section{REFERENCES}

[1] H. Uitenbroek, "Multilevel Radiative Transfer with Partial Frequency Redistribution," Astrophysical Journal, 557, 389-398 (2001).

[2] H. Peter, "On the nature of the transition region from the chromosphere to the corona of the Sun," $A \& A, \mathbf{3 7 4}$, $1108-1120$ (2001).

[3] G. A. Gary, "Plasma Beta above a Solar Active Region: Rethinking the Paradigm," Solar Phys., 203, 71 (2001).

[4] R. Rosner, B. C. Low, and T. E. Holzer, "Physical processes in the solar corona," Physics of the sun, D. Reidel Publishing Co, 135-180 (1986).

[5] Moore, Davis, and Hathaway, "High-Resolution Solar Magnetography from Space: Beyond Solar-B," http://solarscience.msfc.nasa.gov/Beyond Solar-B.shtml, (2001).

[6] J. Davis, E. West, R. Moore, G. Gary K. Kobayashi, J. Oberright, D. Evans, H. Wood, J. L. R. Saba and D. Alexander, "MTRAP: The Magnetic Transition Region Probe," SPIE: Solar Physics and Space Weather Instrumentation. 5901, 1-12 (2005).

[7] Gurman, J. B., "The MG II H line in sunspot umbrae," Solar Phys., 90, 13-15 (1984).

[8] West, E. A., J. G. Porter, J. M. Davis, G. A. Gary, D. M. Rabin, R. J. Thomas, and J. M. Davila, "Overview of the Solar Ultraviolet Magnetograph Investigation," SPIE: Instrumentation for UV/EUV Astronomy and Solar Missions, 4139, 350-361 (2000).

[9] West, E. A., J. G. Porter, J. M. Davis, G. A. Gary, and M. Adams, "Development of a polarimeter for magnetic field measurements in the ultraviolet," SPIE: Polarization Analysis: Measurement and Remote Sensing IV, 4481, pp. 109-117 (2001).

[10] West, E. A., J. G. Porter, J. M. Davis, G. A. Gary, M. Adams, S. Smith and J. F. Hraba, "Optical characteristics of the Marshall Space Flight Center Solar Ultraviolet Magnetograph," SPIE: UV/EUV and Visible Space Instrumentation for Astronmy and Solar Physics, 4498, 101-110 (2001).

[11] K. Kobayashi, E. A. West and M. Noble, "Polarization measurements in the Vacuum Ultraviolet," SPIE: Polarization Science and Remote Sensing II. 5888, 1-12 (2005).

[12] K. Kobayashi, E. A. West, J. M. Davis and G. A. Gary, "Polarization measurements on SUMI's TVLS gratings", SPIE: Polarization Science and Remote Sensing III. 6682, 1-12 (2007).

[13] T. Kita and T. Harada, "Use of Aberration-Corrected Concave Gratings in Optical Demultiplexers," Applied Optics, 22, 819-825 (1983).

[14] R. J. Thomas, "Toroidal varied line-space (TVLS) gratings," SPIE: Innovative Telescopes and Instrumentation for Solar Astorphysics, 4853, 411-418 (2003).

[15] L. Poletto and R. J. Thomas, "Stigmatic Spectrometers for Extended Sources: Design with Toroidal Varied LineSpace (TVLS) Gratings," Applied Optics, 43, 2029-2038 (2004).

[16] NIST, "Platinum Neon Data," http://physics.nist.gov/PhysRefData/platinum/contents.html , 1-2 (2008)

[17] E. A. West, J. G. Porter, J. M. Davis, G. A. Gary, M. W. Noble, M. Lewis and Roger J. Thomas, "The Marshall Space Flight Center Solar Ultraviolet Magnetograph," SPIE: UV and Gamma-Ray Space Telescope Systems, 5488, 801-812 (2004). 